\title{
Simulated Quenching for Cancellation of Non-Linear Multi-Antennas Coupling
}

\author{
Igor Arambasic, Javier Casajus Quiros and Ivana Raos
}

Additional information is available at the end of the chapter

http://dx.doi.org/10.5772/47295

\section{Introduction}

Multiple antennas are a must in today's mobile device providing high data rates and/or large communication range. At the same time, as mobile terminal volume is not enlarged, the level of electronic interferences inside mobile terminals is increased. When observing the flow of data through the terminal energy dissipation of many electronic components interlaced with numerous data metallic wires is detected. Apart from dissipation, all these components also capture some energy dissipated from another source. This undesirable transfer of energy between physical mediums, like metallic wires or circuit segments, is known as coupling. It is likely to occur either in a place where no energy dissipation protection is available due to the lack of space, or where economic electronic elements are used in order to decrease the costs of the product. The interior of mobile receiver terminal fulfils both assumptions and coupling results in an irreducible error floor urging the development of coupling cancellation methods.

In this chapter coupling cancellation module, based on two simulated quenching (SQ) methods, is presented and analyzed. The development of these methods was stimulated by Simulated Annealing (SA) approach which can statistically guarantee finding an optimal solution, as shown in [12], if sufficient number of iterations is available. Since standard SA approach is time-consuming, we decided to ignore physical analogy. Hence, two SQ methods evolved: standard SQ which focuses on precision and Improved Fast SQ where search progress is accelerated on the cost of the decoupling precision.

\section{System description}

\subsection{Non-linear coupling model}

Coupling is commonly defined as undesirable transfer of energy between physical mediums (like metallic wires or optical fibers) or circuit segments. Inside multi-antennas mobile terminal the data received at the antennas is affected on its path both by direct distortion imperfections and coupling as modeled in Figure 1. 


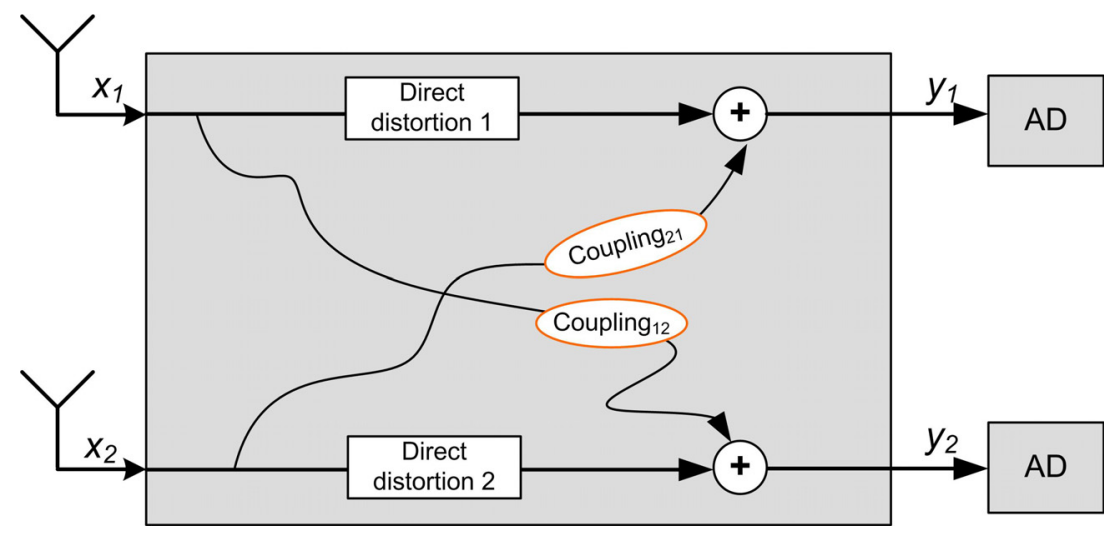

Figure 1. Simplified direct distortion and coupling

In most practical cases the reasonable assumption for the coupling functions is to model them as polynomials and limit them to third order nonlinear behavior which is consistent with the analog electronic elements and follows the nonlinear behavior experienced in many radio frequency (RF) front ends [1]. Hence, if no direct distortion is present, the input of two AD converters can be described mathematically as:

$$
\begin{aligned}
& y_{1}=x_{1}+\left(c_{11} x_{2}+c_{12} x_{2}^{2}+c_{13} x_{2}^{3}\right) \\
& y_{2}=x_{2}+\left(c_{21} x_{1}+c_{22} x_{1}^{2}+c_{23} x_{1}^{3}\right)
\end{aligned}
$$

where $x_{1}$ and $x_{2}$ are the signals received on the corresponding antennas, and $y_{1}, y_{2}$ the signals at the input of $\mathrm{AD}$ converter. Parameters $c_{i j}$ corresponding to the coupling of the $j^{\text {th }}$ order $(j=$ 1..3) experienced on the $i^{t h}(i=1,2)$ input of AD converter signals. These elements describe energy absorption/dissipation that the signals are experiencing when passing through RF front-end. Hence, the direct coupling function is either described mathematically with three parameters $\left(c_{1}, c_{2}, c_{3}\right)$ or as $\left[C_{1}, C_{2}, C_{3}\right]$ in $[\mathrm{dB}]$ describing the physical process of coupling power. The relation between interference amplitude in the coupling function and the interference power at physical level is analyzed in [1] and described as:

$$
C_{i j}=10 \log \frac{c_{i j}^{2} P_{j 0}}{P_{i}}[d B] \Leftrightarrow c_{i j}^{2}=\frac{P_{i}}{P_{j 0}} 10^{C_{i j} / 10}
$$

with $P_{i}$ the power of the received signal at input $i, P_{j 0}$ normalized power of the interference of the $j^{\text {th }}$ order and $C_{i j}$ the coupling parameter expressed in [dB] for the $i^{\text {th }}$ antenna and $j^{\text {th }}$ coupling order. In Table 1 the unit power of three interference orders for different constellations is given. These numbers can be used for simulation purposes when the power of transmitted signal is normalized to 1 .

The influence of coupling, depending on the system performance, can be divided in 3 levels: strong, moderate and weak. The strict definition of these three levels can not be made as it depends on the constellation type. However, the borders can graphically be seen in Figure 2 where the presented curve shows theoretical performance of the corresponding system without coupling. Thus when referred to strong coupling, we are referring to coupling 


\begin{tabular}{|c|c|c|c|}
\hline & $P_{10}, P_{i}$ & $P_{20}$ & $P_{30}$ \\
\hline Binary & 2.00 & 6.00 & 20.00 \\
\hline QPSK & 2.00 & 6.00 & 20.00 \\
\hline 8-PSK & 2.00 & 6.00 & 20.00 \\
\hline 16QAM & 2.00 & 7.92 & 39.20 \\
\hline 64QAM & 2.00 & 7.92 & 39.20 \\
\hline
\end{tabular}

Table 1. The unit power of three interference orders for different constellations

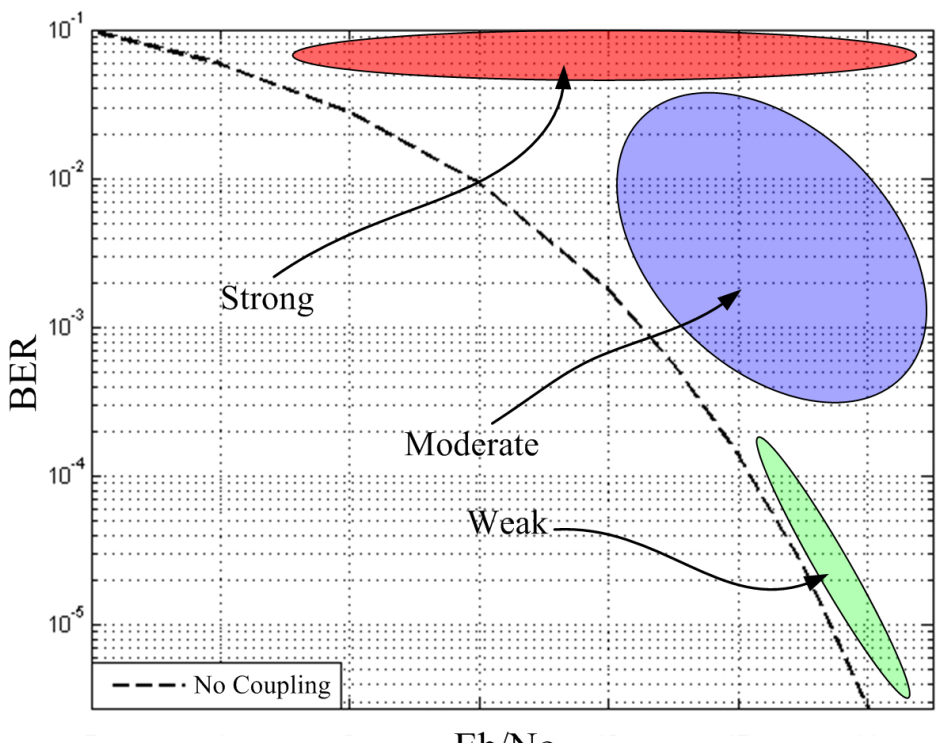

$\mathrm{Eb} / \mathrm{No}$

Figure 2. Coupling strength classification depending on the lost system performance

that almost completely destroys the received signal and whose system performance almost shows flat line. Moderate coupling level follows the theoretical curve, but the system performance exhibits slow improvement, while the weak coupling system follows closely the ideal theoretical curve but the system performance is still visibly deteriorated. In the following, search for the decoupling function is mainly made for strong and medium coupling levels as they are more demanding cases.

\subsection{Coupling cancellation module}

The coupling cancellation is performed with nonlinear software decoupling module located at the output of analog-to-digital (AD) converter as shown in Fig. 3. The advantage of this position is that it deals directly with sampled physical data avoiding any dependency on specific signal type. Inside the module, cancellation is achieved by determining the inverse coupling function. 


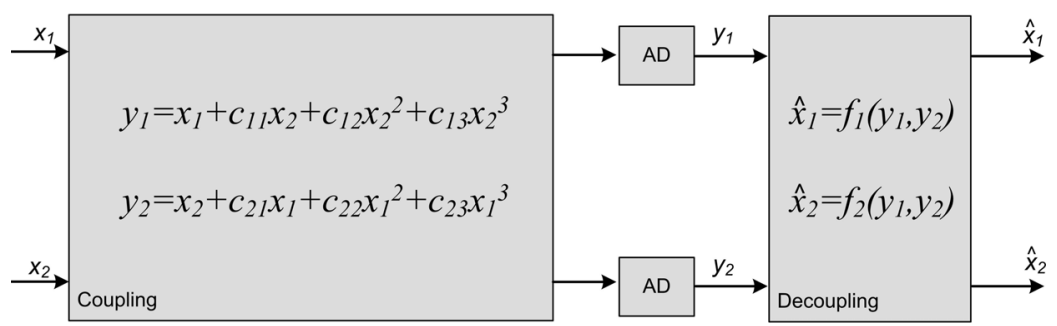

Figure 3. Coupling cancellation module positioned at the output of AD converters

Taking into account that the signals at the output of decoupling module, $\hat{x}_{1}$ and $\hat{x}_{2}$, should theoretically be equal to $x_{1}$ and $x_{2}$, the inverse functions take the form of:

$$
\begin{aligned}
& \hat{x}_{1}=y_{1}-c_{11} \hat{x}_{2}-c_{12} \hat{x}_{2}^{2}-c_{13} \hat{x}_{2}^{3} \\
& \hat{x}_{2}=y_{2}-c_{21} \hat{x}_{1}-c_{22} \hat{x}_{1}^{2}-c_{23} \hat{x}_{1}^{3}
\end{aligned}
$$

Assuming that all distortion parameters $c_{i j}$ are a priori known, a problem of two nonlinear equations with two unknown variables is presented. By extracting $\hat{x}_{2}$ the problem is reduced to single variable and can be described as a nonlinear problem of the ninth order:

$$
\sum_{i=0}^{i=9} k_{i} \hat{x}_{1}^{i}=0
$$

with:

$$
\begin{gathered}
k_{0}=y_{1}-c_{11} y_{2}-c_{12} y_{2}^{2}-c_{13} y_{2}^{3} \\
k_{1}=c_{11} c_{21}+2 c_{12} c_{21} y_{2}+3 c_{13} c_{21} y_{2}^{2}-1 \\
k_{2}=c_{11} c_{22}-c_{12} c_{21}^{2}+2 c_{12} c_{22} y_{2}-3 c_{13} c_{21}^{2} y_{2}+3 c_{13} c_{22} y_{2}^{2} \\
k_{3}=c_{11} c_{23}-2 c_{12} c_{21} c_{22}+2 c_{12} c_{23} y_{2}+c_{13} c_{21}^{3}-6 c_{13} c_{21} c_{22} y_{2}+3 c_{13} c_{23} y_{2}^{2} \\
k_{4}=-2 c_{12} c_{21} c_{23}-c_{12} c_{22}^{2}+3 c_{13} c_{21}^{2} c_{22}-3 c_{13} c_{22}^{2} y_{2}-6 c_{21} c_{23} c_{13} y_{2} \\
k_{5}=-2 c_{12} c_{22} c_{23}+3 c_{13} c_{21} c_{22}^{2}+3 c_{13} c_{21}^{2} c_{23}-6 c_{13} c_{22} c_{23} y_{2} \\
k_{6}=-c_{12} c_{23}^{2}+6 c_{13} c_{21} c_{22} c_{23}+c_{13} c_{22}^{3}-3 c_{13} c_{23}^{2} y_{2} \\
k_{7}=3 c_{13} c_{21} c_{23}^{2}+3 c_{13} c_{22}^{2} c_{23} \quad k_{8}=3 c_{13} c_{22} c_{23}^{2} \quad k_{9}=c_{13} c_{23}^{3}
\end{gathered}
$$

It is shown in [1] that the existence of one analytically expressed inverse function supporting the range of all input pairs $\left(x_{1}, x_{2}\right)$ is extremely low and depends on all involved parameters $\left(x_{1}, x_{2}, c_{11}, c_{12}, c_{13}, c_{21}, c_{22}, c_{23}\right)$, together with the signal constellation definition which determines the root space. Consequently mathematical surface approximation in combination with calibration process is proposed.This approach is based on finding a surface that matches a series of constraints by minimizing the distance between reference and re-constructed or inverse surface. In other words, a set of predefined points, denominated calibration data, are available to the observed process (in our case coupling) forming the 
reference surface. This reference surface is then used as basis for building the approximated inverse surface.

With the introduction of the fitting surface approach the solution to the inverse coupling problem can be divided into two steps: calibration process and point-to-point real-time decoupling. Once all decoupling surface design parameters are defined, the decoupling, based on eq. 9, is implemented inside coupling cancellation module on all incoming pair of signals achieving signal enhancement with very low processing demands.

In order to develop a surface fitting algorithm four important parameters should be defined, namely:

- calibration data

- mathematical surface model

- cost function

- parameter search algorithm

\subsubsection{Calibration data}

The calibration process includes the transmission of the calibration data used for the reference surface construction and the search for the inverse surface based on some search optimized algorithm. The calibration data should be well defined covering the whole direct function domain. For discrete signal coupling systems, the calibration points would correspond to the constellation specific points of the two antennas. In other words the whole domain of two 16 QAM constellations is covered with at least $256(16 \times 16)$ data points, while 64 QAM constellation requires a minimum of $4096(64 \times 64)$ points as the influence of all point combinations should be analyzed. The required number of calibration points is analyzed in detail in [1]. All the results presented in this chapter correspond to 2000 calibration symbols.

\subsubsection{Mathematical surface model}

Low-order polynomials tend to be smooth and high order polynomial curves like the one of the ninth degree, tend to be "lumpy" with several local minimum and maximums making the surface approximation severe. Having in mind that complex curves can be formed without higher order multiplications, a model based on a sum of two independent higher order polynomials with one simple linear multiplication is proposed in [3]:

$$
\hat{x}_{1}=\sum_{i=1}^{i \leq N_{1}} a_{i} y_{1}^{i}+\sum_{j=1}^{j \leq N_{2}} b_{j} y_{2}^{j}+k_{1} y_{1} y_{2}+k_{2}
$$

where $N_{1}$ and $N_{2}$ represent the degrees of freedom of variables $y_{1}$ and $y_{2}$ respectively, while variables $a_{i}, b_{j}, k_{1}, k_{2}$ are the decoupling coefficients that are to be found with surface approximation search.

This surface function is simple and offers a unique solution to each input pair. The ranks of polynomials, controlled with $\left(N_{1}, N_{2}\right)$, are determined as a trade off between the acceptable system performance and the required computational power. In [2], the approximation with 20 coefficients for the coupling cancellation fitting surface is proven to be accurate enough. 
Hence, $\left(N_{1}, N_{2}\right)=(9,9)$ is selected for describing the mathematical surface model. In order to be able to adopt the surface search method to general decoupling problem, the data, on which the search is carried out, is restrained to $\langle-1,1\rangle$. This is achieved by implementing the normalization according to the maximum received symbol level taking into account all calibration symbols on both antennas, as shown in the following equation:

$$
\begin{aligned}
& y_{1 k}^{N}=\frac{y_{1 k}}{\operatorname{Max}\left(\left|y_{1 k}\right|,\left|y_{2 k}\right|\right)} k=1 . . N \\
& y_{12 k}^{N}=\frac{y_{12 k}}{\operatorname{Max}\left(\left|y_{1 k}\right|,\left|y_{2 k}\right|\right)}
\end{aligned}
$$

where $y_{1 k}^{N}$ and $y_{2 k}^{N}$ are the $k$-th normalized received symbols on the corresponding antennas, $\operatorname{Max}\left(\left|y_{1 k}\right|,\left|y_{2 k}\right|\right)$ is the maximum received absolute value level, and $N$ is the number of calibration signals. Eventually, since the approximation surface is constructed based on the normalized levels, the true value of reconstructed symbol is obtained by expanding the reconstructed normalized symbol $\hat{x}_{1 k}^{N}$ to the received dynamic range:

$$
\hat{x}_{1 k}=\hat{x}_{1 k}^{N} \operatorname{Max}\left(\left|y_{1 k}\right|,\left|y_{2 k}\right|\right)
$$

Hence, the decoupling approximation surface is defined as:

$$
\hat{x}_{1 k}=\operatorname{Max}\left(\left|y_{1 k}\right|,\left|y_{2 k}\right|\right)\left\{\sum_{i=1}^{i \leq N_{1}} a_{i}\left(y_{1 k}^{N}\right)^{i}+\sum_{j=1}^{j \leq N_{2}} b_{j}\left(y_{2 k}^{N}\right)^{j}+k_{1} y_{1 k}^{N} y_{2 k}^{N}+k_{2}\right\}
$$

This way, the processing load of the decoupling process is basically concentrated on the approximation fitting surface parameter search.

\subsubsection{Cost function}

The objective of surface search consists in finding the decoupling coefficients that minimize the cost function. The calculation of cost function has to be carried out for each new candidate and presents the most demanding part of the decoupling processing load. The cost function typically implemented for the search of approximate inverse function is root mean square, also known as the quadratic mean:

$$
Q_{j}=\sqrt{\frac{1}{N} \sum_{i=1}^{N}\left(x_{j i}-\hat{x}_{j i}\right)^{2}}
$$

where $N$ is the number of symbols used, $x_{j i}$ is the sent $i^{\text {th }}$ calibration symbol on $j^{\text {th }}$ antenna, and $\hat{x}_{j i}$ its corresponding value calculated using the coefficients of new surface candidate according to eq. 9. The set of decoupling coefficients displaying minimum $Q_{j}$ value are then used in decoupling module as the inverse coupling surface function.

\subsubsection{Parameter search algorithm}

SA methods are chosen as starting point since this approach can statistically guarantee finding an optimal solution, although no estimate can be made on the number of trials needed to reach it. The name and inspiration come from the annealing in metallurgy, as the technique mimics 
the nature of the metal which is heated and than cooled slowly in order to reach its absolute minimum energy state.

During the last few decades a number of variations of original SA algorithm have been published. The comparison of different SA algorithms, with other mostly heuristic algorithms can be find in $[4,6-10,13,18]$. Regardless of different variations, any algorithm based on physical SA has proven to be an effective global optimization method because of several important features:

- it can process the cost functions with arbitrary degrees of nonlinearities and discontinuities, as no restriction on the form of the cost function exists

- it can statistically guarantee finding an optimal solution

- it can be implemented easily when convergence speed is not relevant

The basic assumption of the SA class of approaches is that sometimes, in order to avoid the trap of local minimum, intermediate points that lead to worse minimum solution are accepted. The fact is that classical SA search is very slow when compared to other heuristic methods and is often avoided as more importance is given to speed than to precision. Hence, the original SA algorithm is rarely used, but it is worth mentioning some of the known SA enhancements like Quantum Annealing (QA), Simulated Quenching (SQ), Mean Field Annealing (MFA), Fast Simulated Annealing (FSA), Parallel Recombinative Simulated Annealing (PRSA) or Adaptive Simulated Annealing (ASA) that have been proposed in numerous articles [5, 7, 14-16]. In contrast to the original SA approach these methods have been characterized as fast search algorithms. Eventually, inspired by SA algorithms two SQ approaches, which do not follow physical analogy, are put forward for parameter search algorithms. The first one offers high precision but requires large number of iterations to reach it, while inside the second one the search progress is accelerated on the cost of the decoupling precision. Still, the decoupling results are kept at high level which make the precision loss acceptable compared to the speed gain.

\section{Simulated quenching applied to nonlinear decoupling problem}

The functioning of the classical SQ method can be described using the pseudo code, divided into initialization and the simulated annealing part as presented in Figure 4.

Inside the first section the temperature factor $T$ is set to the initial temperature $\left(T_{0}\right)$. The temporary minimum of the cost function $\left(Q_{\min }\right)$ is calculated for the initial set of decoupling coefficients $\left(\operatorname{Coef}_{0}\right)$. These values are then appointed to actual state configuration. Afterwards, the algorithm enters the search loop until allowed processing time measured in number of iterations is exceeded (Iter $>I_{M A X}$ ). The selection of new coefficients (neighbors) is done inside the New_Candidate function, and then the cost function for the set of selected coefficients is calculated $\left(Q_{\text {new }}\right)$. If the cost function (Cost) of the new neighbor presents lower value than temporary minimum cost function $\left(Q_{\min }\right)$, the perturbation is accepted. The new cost function value $\left(Q_{\text {new }}\right)$ becomes the new minimum and the corresponding set of the coefficients is stored in Coef $_{\text {min }}$. In case of higher cost function value the perturbation is accepted only if the randomly selected number between zero and one is smaller than the Trans_Prob function of the actual temperature T. However, in this case only the actual position is updated and the global minimum cost configuration is left unchanged. The search at one temperature level is limited 


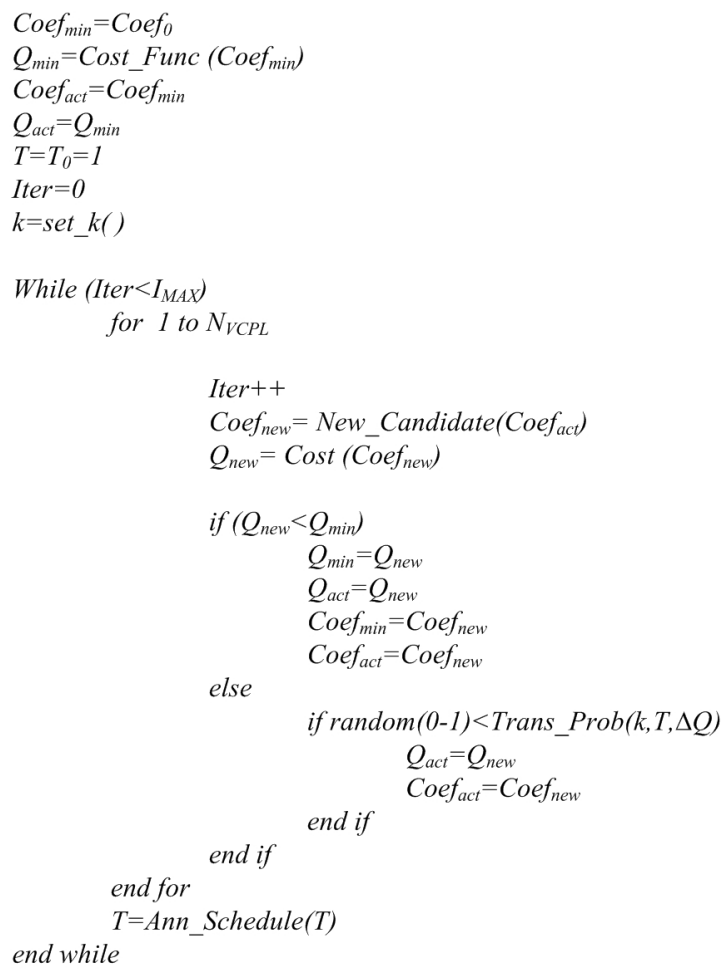

Figure 4. Pseudo code of SQ applied to Nonlinear Decoupling Problem

with a number of visited candidates per level defined with $N_{V C P L}$ parameter. Afterwards, the temperature $T$ is decreased according to the annealing schedule function (Ann_Schedule) and the quest is continued.

According to the formulation of SQ algorithm when applying the SQ method to a decoupling problem, one must specify:

- the state space or the coefficient resolution

- symbol precision $\left(\operatorname{Res}_{\text {symb }}\right)$

- coefficient precision ( Res $_{\text {coef }}$ )

- the initial coefficients values or the starting point $\left(\right.$ Coef $\left.f_{0}\right)$

- the neighbor selection method (New_Candidate) - coefficient maximum displacement $\left(R_{0}\right)$

- the form of the cost function (Cost_Func) - already defined with Equation 10.

- the probability transition function (Trans_Prob) - initial (maximum) probability of the acceptance of the worse move ( $\left.p_{\max }\right)$

- the annealing schedule function (Ann_Schedule)

- time reduction factor $(\alpha)$

- number of visited candidates per temperature level $\left(N_{V C P L}\right)$ 
- maximum allowed number of iterations $\left(I_{M A X}\right)$

- minimum probability of worse move during the search $\left(p_{\min }\right)$

\subsection{Tuning of SQ parameters}

The choice of SQ parameters can have a significant impact on the method effectiveness. Unfortunately, there is no selection good enough for all problems, and there is no general way of finding the best set of parameters for a given problem. However, some more generic parameters (e.g. the annealing schedule or probability transition function) can be set based on the experience of other authors like $[8,9,11,13,14,16]$. This way, preliminary SQ search executions are over-dimensioned favoring precision over speed and are used for tuning correctly the search parameters. The empirical tuning of SQ parameters is done for 64QAM signals. The results are based on 2000 calibration symbols transmitted under SNR of 100dB. This way the analysis is concentrated primarily on coupling rather than on noise effects. Since SQ search carries probability factor each presented simulation is in fact executed ten times, and the figures present the averaged values.

\subsubsection{The state space}

This parameter is defined with the number of design variables, their discrete domain resolution and the overall symbol precision. Starting from a analog domain the search coefficients are transformed into discrete domain by setting the distance between two sample points. For example, by setting the resolution to 0.1 the coefficients can only obtain resolution multiples values like $0.2,-0.1,0.8$ etc. By setting the resolution to more dense value, like 0.0001, exactly 1000 times more possible candidates are available for each coefficient inside the SQ search. Expanding the solution search area naturally leads to better solution precision, but at the expense of processing time. Apart from the coefficients resolution, the state space also defines the resolution of the received and decoupled symbols which depend on the transmission system requirements and implemented A/D converter. We propose to set $\operatorname{Res}_{\text {symb }}=0.00001$ and apply the same resolution on decoupling symbols, that is, $\operatorname{Res}_{\text {coef }}=0.00001$.

\subsubsection{Coefficients initial values}

The initial assumption is that no coupling takes place, and the signal arrives without any distortion. Consequently, the signals that suffered larger distortions will require longer search time as their solution is located far from the starting point. This way the algorithm favors the solutions of smaller coupling which is expected to occur more frequently under realistic conditions. By using the decoupling model in accordance to eq. 9, all coefficients are set to zero, except $a_{1}$ which is set to one:

$$
\text { Coef } f_{0}:\left\{\begin{array}{l}
a=[1,0,0,0,0,0,0,0,0] \\
b=[0,0,0,0,0,0,0,0,0] \\
k=[0,0]
\end{array}\right.
$$

In fact, to make the indexing simpler, the coefficients are expressed as 20 dimensional vector where first nine elements correspond to coefficients $a_{i}, i=1, . ., 9$, second nine elements correspond to $b_{i}, i=1, . ., 9$, and the last two correspond to $k_{1}$ and $k_{2}$. 


\subsubsection{Probability transition function}

The key SA feature that prevents the system of becoming stuck in a local minimum is its probability to move to the new state even when this new state has worse characteristics than the current one. The probability of making the transition from the current state to a candidate new state is a function of the energies of the two states, and of a global time varying parameter $T$ denominated as temperature. Even though the algorithm is open to the use of any probability function $(p)$, the new configurations are usually accepted or rejected according to the Boltzmann probability distribution, $k_{B}$ is the Boltzmann constant that relates temperature to energy. When SA problem is not related directly to the physical energy this constant is replaced with problem specific constant $K$ and modified Boltzmann probability function is used accordingly:

$$
p(\Delta E)=e^{-\frac{\Delta E}{k_{B} T}} \longleftrightarrow p(\Delta Q)=e^{-\frac{\Delta Q}{K T}}=e^{-\frac{\text { Qnew }-Q_{\text {act }}}{K T}}
$$

where $Q_{\text {new }}$ and $Q_{a c t}$ are the cost function values associated with the actual and new candidate state respectively, and $\Delta Q$ their difference. However for a given $\Delta Q$ the transition probability is not constant throughout the annealing process as it also depends on the actual temperature. By setting the initial temperature $T_{0}$ to high values the probability of search wandering is increased. For example, if $\Delta Q=0.1$ and initial temperature $K T_{0}=1$ the perturbation will be accepted if the random variable falls between $\langle 0,0.9\rangle$ which correspond to $90 \%$, while for $\Delta Q=0.1$ and initial temperature $K T_{0}=0.1$ this probability falls to $36 \%$. As the temperature is constantly decreased according to the annealing schedule, the probability of worse move tends to zero. This is in accordance with the physical simulated annealing where no wondering around is possible at low temperatures.

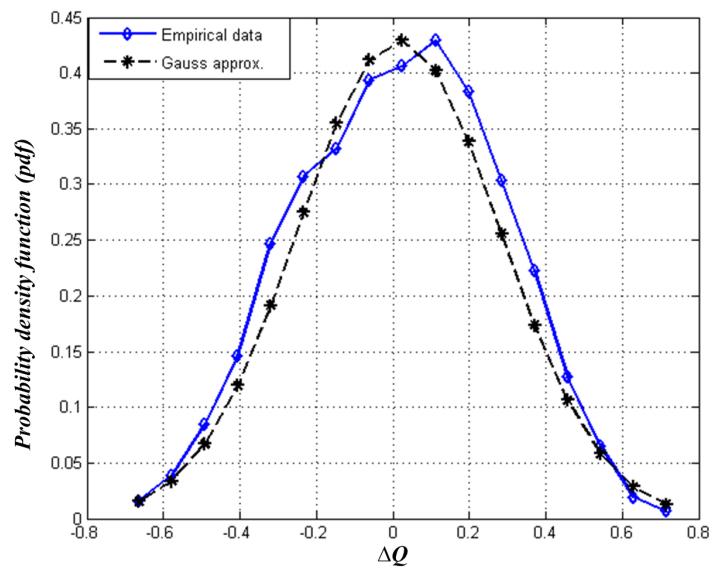

Figure 5. Approximation of the consecutive cost function differences with Gauss function

The selection of problem specific constant $K$ depends on the initial probability of uphill movement acceptance defined as $p_{0}$ which should be guaranteed for wide range of energy differences. In order to define this energy level we approximated the cost differences of the two consecutive surfaces $(\Delta Q)$ with Gaussian probability function, as it fits the actual empirical data. This is shown in Figure 5 where 2000 differences are calculated with maximum 
allowed individual coefficient displacement of $R_{0}=0.05$. By approximating $\Delta Q$ distribution with Gaussian-like probability density function, three standard deviations $\left(\sigma_{\Delta Q}\right)$ represent $99.7 \%$ of all possible cost function differences and as $p_{0}$ can be created according to this value as:

$$
p_{0}=p_{\max }=e^{-\frac{3 \sigma_{\Delta Q}}{K T_{0}}}
$$

with $T_{0}=1, K$ is obtained as:

$$
K=-\frac{3 \sigma_{\Delta Q}}{\ln \left(p_{\max }\right)}
$$

By setting the initial probability of uphill movement close to one, the initial search wander is allowed regardless of the energy difference between two candidates. This slows down the search usually without any solution precision gained. Hence, the initial probability level is generally set to $p_{\max }=50 \%$ and numerical value of $K$ is calculated in $\operatorname{set}_{K}$ function before algorithm enters SQ search loop. Inside the $\operatorname{set}_{K}$ function the approximation of Gauss distribution is obtained by implementing the actual neighbor selection method on 2000 new candidates.

\subsubsection{Annealing schedule function}

The annealing schedule function has no strict definition, as the function only restricts to uphill surface exploration at the beginning, and then gradually restraining the search wondering favoring the movement in the direction of better solution only. Inside physical simulated annealing the probability of acceptance of uphill movement is directly related to temperature, but in mathematical interpretations the initial temperature level has lost its significance, and thus it is set to $T_{0}=1$ in order to simplify the calculation process. Consequently, modified Boltzmann constant $K$ is calculated accordingly. A popular choice for the annealing function is the exponential schedule, where the temperature level, denoted as $T$ or $T_{L}$, is decreased by a fixed factor $\alpha$ :

$$
T_{L}=\alpha T_{L-1} ; T_{0}=1,0<\alpha<1, L=1,2,3 \ldots
$$

where $T_{L}$ and $T_{L-1}$ are the new and the actual temperature respectively, and index $L$ stands for the number of temperature level changes.

In order to define the annealing schedule both, temperature reduction factor $\alpha$ and number of visited candidates per level $N_{V C P L}$ have to be determined. In theory, when keeping $N_{V C P L}$ constant, the search is done more thoroughly if the temperature is decreased slower and better solution precision is achieved. Additionally, if $(\alpha)$ is constant, and $N_{V C P L}$ is increased, each level is scanned in more detail and, again, better results are expected. However, when both factor are set in favor of precision the search is extremely slowed down and the precision improvement might not justify the processing load.

These two parameters are established empirically. In Figure 6 the dependence of the cost function minimum on the number of visited points per level is shown, with bars of different colors corresponding to different temperature reduction factors. The simulations are done for moderate coupling distortion of $[-10,-12,-12] \mathrm{dB}$ and maximum allowed displacement set to $R_{0}=0.05$. It is seen that as $\alpha$ approaches 1 the probability of finding the better solution increases, and as a result minimum cost function moves away from its starting value. However, if $N_{V C P L}$ is low, e.g. $N_{V C P L}=2$ no significant progress is made even with $\alpha=0.995$. This is due to the scan space around the actual minimum which is always kept narrow and so 


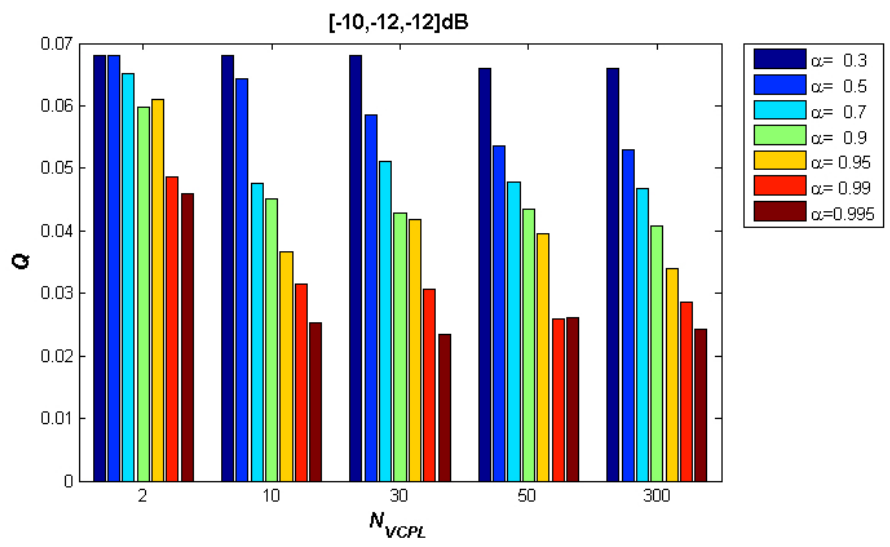

Figure 6. $Q$ as a function of $N_{V C P L}$ and $\alpha$ for [-10,-12,-12] dB coupling distortion

the search is easily stacked in the local minimum. According to empirical decoupling results, the number of visited points per level starts producing good results with $N_{V C P L} \geq 10$.

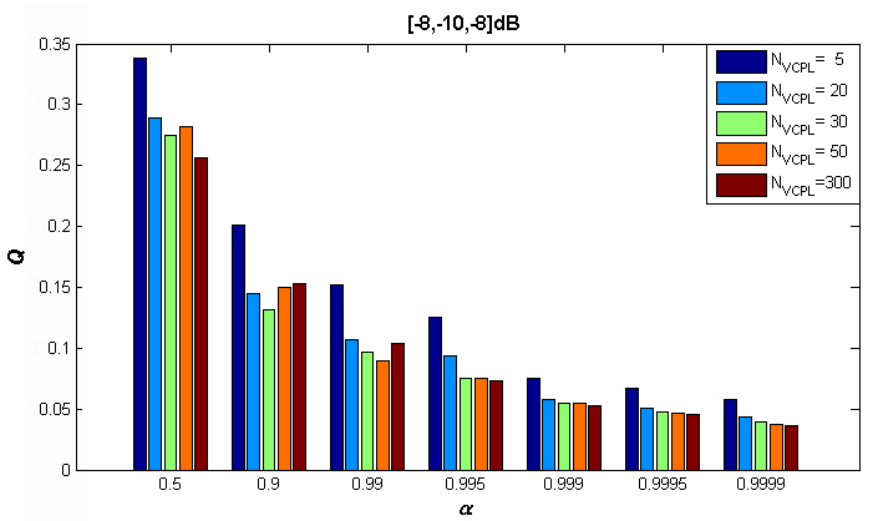

Figure 7. $Q$ as a function of $\alpha$ and $N_{V C P L}$ for $[-8,-10,-8] \mathrm{dB}$ coupling distortion

The cost performance as a function of temperature reduction factor is shown in Figure 7 where bars of different colors correspond to different $N_{V C P L}$ levels. For $\alpha$ between 0.5 and 0.95 the search progress presents low consistency as the cost function minimum experienced big differences between two realizations of the same SQ search. It shows that the temperature reduction is reduced too quickly and that the final precision is still not guaranteed as search robustness is not achieved. This statement is confirmed in Figure 7 by comparing the $Q$ function obtained with different $N_{V C P L}$ for this $\alpha$ range. For $\alpha=0.9$ better precision is achieved with $N_{V C P L}=30$ than with $N_{V C P L}=300$. Hence in order to avoid the uncertainty, temperature reduction factor should be between 0.95 and 1 since this area shows signs of consistency with theoretical background (precision is increased with the increase of either $N_{V C P L}$ or $\alpha$ ). According to the presented simulation results, the SQ search is adopted adequately to inverse coupling approximation with the number of visited candidates per level set to $N_{V C P L}=30$, and temperature reduction factor set to $\alpha=0.995$. 


\subsubsection{Neighbor selection method}

When selecting new candidates (neighbors), it must be possible to move from the initial state to a good enough state by relatively short path while at the same time allowing the search to scan the area but never loosing the good path from the sight. The selection of the new candidate set $\left(\right.$ Coef $\left.f_{\text {new }}\right)$ can be described as:

$$
\operatorname{Coef}_{\text {new }}(i)=\xi_{i} R_{0}+\text { Coef }_{\text {act }}(i) ; i=1, \ldots, 20
$$

where $\xi_{i}$ is the randomly chosen number between -1 and 1 , and $R_{0}$ defines maximum allowed coefficient displacement. If $R_{0}$ is large, the SQ search can easily explore wide search area, but needs more iterations to reach the optimal solution as the algorithm is easily distracted and moved away from the good path. If $R_{0}$ is small, the algorithm can get stuck in local minimum and conclude the search far from the optimal solution.

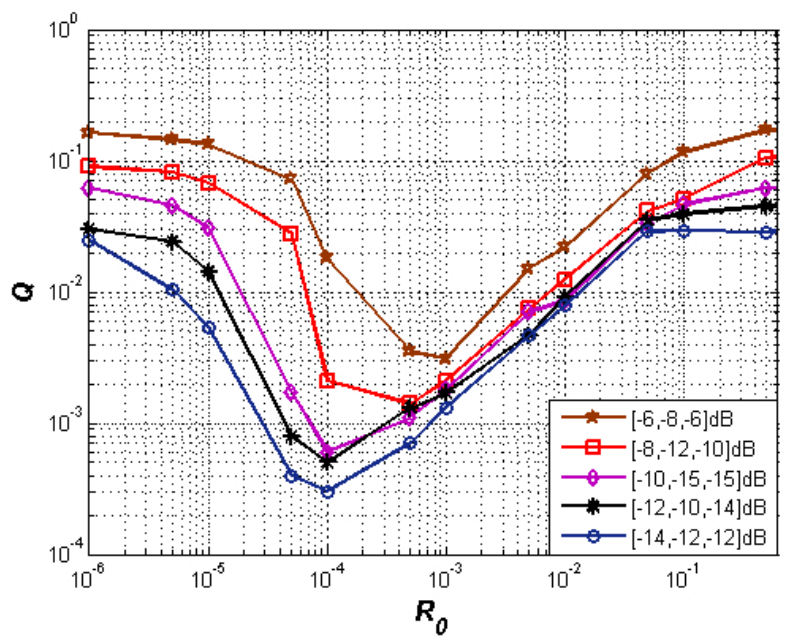

Figure 8. $Q$ in dependence of $R_{0}$ under different coupling conditions

The behavior of cost function with respect to $R_{0}$ under different coupling conditions is presented in Figure 8 as this parameter is retrieved empirically. The conducted search is based on classical SQ algorithm with $N_{V C P L}=30$ and $\alpha=0.995$. The figure can be divided into three clearly distinguished areas. The left side, with $R_{0}$ lower than $10^{-5}$ corresponds to algorithm where no wandering around is allowed. The search progress is slow and any encountered local minimum is presented as the final solution. The optimal solution is only found if the search is executed close to it. On the right side of the figure with $R_{0}$ values larger than 0.5 , wandering is allowed during the whole search process and as a consequence no clear search path is established. The search tends to move too quickly and without any exploration around the actual position. As search is randomly wandering around, finding the optimal solution in this area is highly uncertain. The central part of the image coincides with the meaningful SQ search where wandering around and detail space exploration are in balance. Here, the search method does follow some path and the traps of local minimums are successfully avoided. Based on the presented $Q$ values, we propose to set maximum allowed displacement to $R_{0}=0.0005$. 


\subsubsection{Maximum number of iterations ( $\left.I_{M A X}\right)$}

The strength of SQ method lies in its ability to statistically deliver a true global minimum. However, as mentioned, one of the drawbacks of this method is its slow delivery of the ideal solution once the global minimum area is located. As no indication of time required for reaching the optimal solution is available, the search process is typically suspended after the allowed search time is spent or the cost function has reached the desired level of precision.

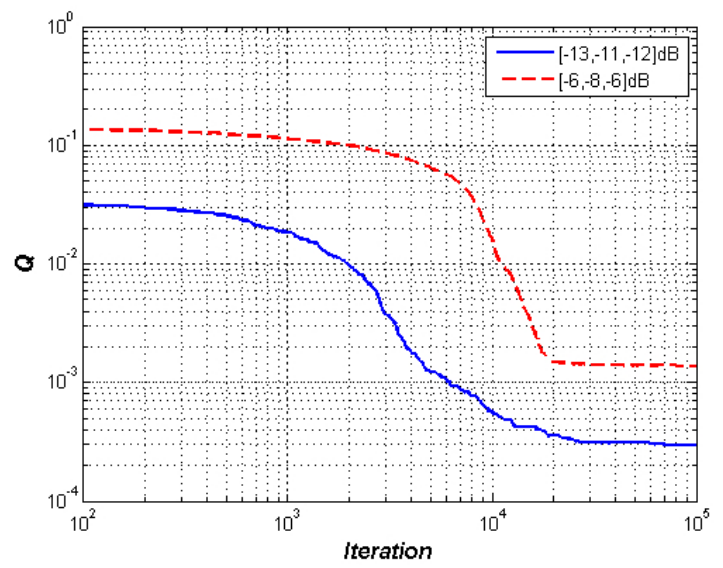

Figure 9. $Q$ in dependence of the number of iterations under two different coupling levels

In Figure 9, $Q$ is depicted as a function of the number of iterations under two different coupling conditions. Both $Q$ functions experience three different segments which correspond to surface wandering at the beginning, almost downhill movement in the middle, and error floor at the end. In both cases error floor is reached after around 20000 iterations and it would make sense to use this number as maximum number of permitted iterations. Still, if we take into account that surface with $Q$ level smaller than 0.005 produce gratifying decoupling results, in this cases the search can be stopped after 4000 and 12000 iterations respectively. The process of establishing the satisfying level of cost function $\left(Q_{s u f f}\right)$ is explained in [1] where relation between BER and the corresponding quadratic mean square cost function level is analyzed. In the end we propose to set maximum number of iterations to 16000 and use it in combination with satisfying cost function level to control the search execution.

\subsection{SQ example}

The SQ search is conducted under moderate coupling conditions of $[-10,-12,-10] \mathrm{dB}$ using the SQ search parameters adopted to nonlinear decoupling problem gathered inside Table 2. The table consist of two columns with standard values based on theoretical assumptions occupying the left column, and values based on empirical decoupling analysis in the right column.

The behavior of adopted SQ search algorithm is shown in Figure 10, where cost function is presented in dependence of number of iterations for 10 different SQ search executions. The image shows that the search path in first 500 iterations is similar in all ten instance. The curves then start to spread, and are grouped again at the end. Nevertheless, the cost 


\begin{tabular}{|c|c|}
\hline Standard Values & $\begin{array}{c}\text { Values based on Empirical } \\
\text { Decoupling Analysis }\end{array}$ \\
\hline Res $_{\text {symb }}=0.00001$ & $N_{V C P L}=30$ \\
\hline $\operatorname{Res}_{\text {coeff }}=0.00001$ & $\alpha=0.995$ \\
\hline Coef $_{0}(i)=\left\{\begin{array}{l}1, \quad i=1 \\
0, i=2, \ldots, 20\end{array}\right.$ & $R_{0}=0.0005$ \\
\hline$Q=\sqrt{\frac{1}{N} \sum_{i=1}^{N}\left(x_{1 i}-\hat{x}_{1 i}\right)^{2}}$ & $Q_{\text {suff }}=0.005$ \\
\hline $\begin{array}{c}\text { Coef } f_{\text {new }}(i)=\xi_{i} R_{0}+\operatorname{Coef}_{\text {act }}(i) \\
i=1 . .20 ; \xi_{i} \in\langle-1,1\rangle\end{array}$ & $I_{M A X}=16000$ \\
\hline$p(\Delta Q)=e^{-\frac{\Delta Q}{k T}}$ & \\
$k=-\frac{3 \sigma_{\Delta Q}}{\ln \left(p_{\max }\right)}$ & \\
\hline$T_{L}=\alpha T_{L-1}$ & \\
\hline$T_{0}=1, \alpha<1, L=1,2,3 \ldots$ & \\
\hline$p_{\max }=50 \%$ & \\
$p_{\min }=0.1 \%$ & \\
\hline
\end{tabular}

Table 2. Set of SQ search parameters after empirical analysis

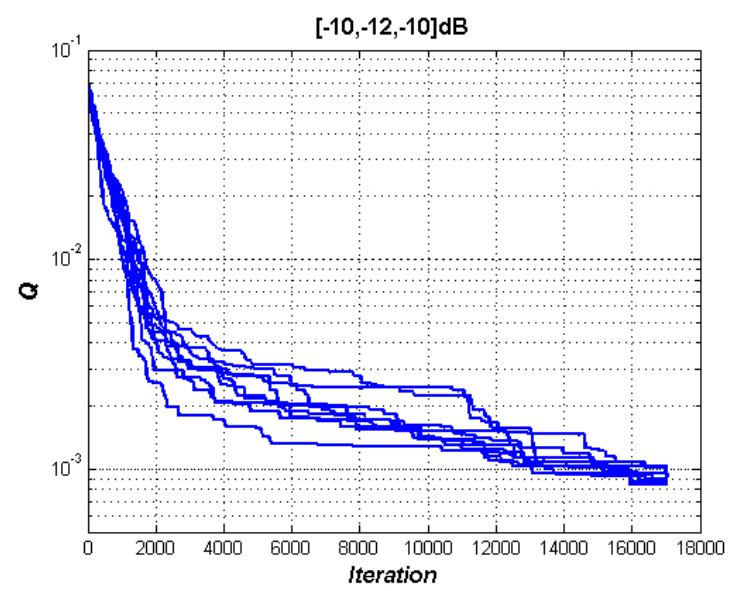

Figure 10. Ten instances of adopted SQ search algorithm under moderate coupling

function solutions are kept in the same orders of magnitude throughout the entire search process. The small differences between the temporary cost function progress and stable final solution precision confirms that the SQ parameters have been selected adequately. The search successfully avoids local minimum traps and follows the correct search path. Furthermore, since BER performance enters saturation for $Q<0.005$ the presented search can be stopped at 4000 iterations preserving the decoupling precision.

Even though the obtained cost function value is similar for all ten instances of conducted SQ search, the presented decoupling coefficients numerically offer completely different solutions. Two different sets of 20 decoupling coefficients, obtained as a result of two execution of classical SQ algorithm under the same coupling conditions, are presented in Figure 11. The difference between the cost functions in this case is almost zero as surface cost functions are 


\begin{tabular}{|c|c|c|c|c|c|c|c|c|}
\hline a1 & a2 & a3 & 24 & 25 & a6 & a7 & a8 & a9 \\
\hline 1,00840 & 0,00468 & 0,00433 & 0,00218 & 0,00245 & $-0,02087$ & 0,00018 & 0,01541 & $-0,00384$ \\
\hline$b_{1}$ & $b_{2}$ & $b_{3}$ & $b_{4}$ & b5 & b6 & $b_{7}$ & b8 $_{8}$ & b9 \\
\hline$-0,09903$ & $-0,01802$ & $-0,01042$ & $-0,00752$ & 0,00664 & 0,02397 & $-0,01103$ & $-0,01454$ & 0,00752 \\
\hline k1 & $k_{2}$ & & & & & & & \\
\hline 0,00237 & 0,00002 & & & & & & & \\
\hline & & & & & & & & \\
\hline a1 & $a_{2}$ & $a_{3}$ & $a_{4}$ & a5 & a6 & a7 & as & a9 \\
\hline 1,01160 & $-0,00424$ & 0,00040 & 0,00448 & $-0,01285$ & 0,01531 & 0,01581 & $-0,01373$ & $-0,00417$ \\
\hline$b_{1}$ & $b_{2}$ & $b_{3}$ & $b_{4}$ & b5 & b6 & $b_{7}$ & $b_{8}$ & b9 \\
\hline$-0,10036$ & $-0,01088$ & $-0,00315$ & $-0,00896$ & $-0,01443$ & $-0,00261$ & 0,01502 & 0,00645 & $-0,00351$ \\
\hline k1 & k2 & & & & & & & \\
\hline 0,00298 & $-0,00001$ & & & & & & & \\
\hline
\end{tabular}

Figure 11. Two sets of decoupling coefficients for $[-10,-12,-10] \mathrm{dB}$ coupling environment

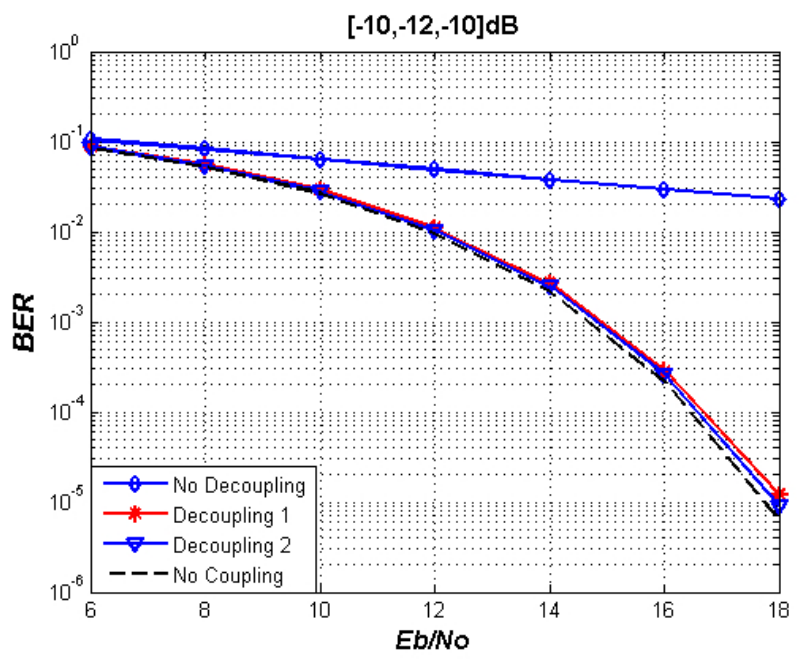

Figure 12. Performance of SQ based decoupling module under moderate coupling

$Q_{a}=0.0009$ and $Q_{b}=0.0008$ respectively. This points out that the constructed inverse coupling surfaces are similar. However, they are based on different building material as for example $a_{7}$ is very close to zero in first solution while in second one is $a_{7}=0.015$. On the other hand $a_{5}$ and $b_{3}$ show opposite behavior, as they present higher importance in second than in first solution. Furthermore, the amplitudes of decoupling coefficients don't exhibit the tendency of reduction as the exponential order is increased. For example, $a_{8} \gg a_{3}$ or $b_{7} \gg b_{3}$ indicating that the surface simplification based on simple reduction of the order of polynomials might not achieve the same decoupling efficiency.

The system performance of decoupling module based on the obtained coefficients traces almost the same curve inside $E_{b} / N_{0}$-BER graph as shown in Figure 12. The entire description of system used for transmission simulations is found in [1]. The upper curve on the image presents the system behavior under coupling of $[-10,-12,-10] \mathrm{dB}$ and without decoupling module. The lowest curve shows the theoretical system performance without any distortion and only with additive white Gaussian noise (AWGN). The two curves in the middle show that the system performance with the decoupling module is very close to the theoretical system performance This confirms that the cost function and inverse surface model are both selected adequately and that SQ search produces accurate results. 


\section{Improved Fast Simulate Quenching (IFSQ)}

The proposed Improved Fast SQ (IFSQ) approach applies changes to three vital parts of SQ method:

- annealing schedule

- probability transition function

- maximum allowed displacement.

The IFSQ implements the annealing schedule function according to Boltzman function while the probability transition function corresponds to Caucy distribution. The novel part of IFSQ approach is also to keep the maximum allowed displacement factor a function of temperature.

All enhanced SA methods labeled as fast are based on steeper annealing schedule function when compared to traditional SA approach. Previously described SQ approach is based on the exponential annealing schedule function where the temperature level is decreased by a fixed factor during the entire search process. However, it makes sense to decrease the temperature more rapidly at the beginning when wandering is desirable, and than reduce the temperature carefully towards the end when SQ turns into a greedy search algorithm. This is precisely the effect achieved with the following annealing schedule function used in IFSQ approach:

$$
T_{L}=\frac{T_{\alpha}}{\ln (L+1)} ; \quad L=1,2,3 .
$$

where $L$ is the actual temperature level and $T_{\alpha}$ is the temperature constant. The numerical value of $T_{\alpha}$ is actually irrelevant to performance of IFSQ search. In real physical process this factor controls the initial temperature and as such influences the probability of uphill movement during the search since probability is directly related to temperature with Boltzmann constant $k_{B}$. As decoupling problem is not related directly to the physical energy $k_{B}$ is replaced with problem specific constant $k_{c}$ which is adjusted according to the initial temperature level. In order to easily compare the SQ to IFSQ search $T_{\alpha}$ is chosen to equal the initial temperature level of SQ search algorithm:

$$
\alpha T_{0}=\frac{T_{\alpha}}{\ln (2)} \quad \Rightarrow \quad T_{1}^{S Q} \quad T_{\alpha}=\alpha \ln (2)
$$

with $T_{0}=1$ and $\alpha=0.995$ which corresponds to the temperature reduction factor of the adjusted SQ search. As a result, the initial temperature level of IFSQ method is set to $T_{\alpha}=0.69$.

The difference between SQ and IFSQ annealing schedules based on adjusted parameters is seen in Figure 13. As can be seen the temperature level of the SQ algorithm is kept high during the first ten levels. At this time, IFSQ temperature is decreased to less then $30 \%$ of the initial temperature and shortly afterwards starts to decrease slowly, while SQ temperature decrease now gets steeper. At the end, IFSQ keeps temperature almost constant allowing the thorough search of actual neighbor space. On the other hand, SQ search turns into a greedy algorithm without any uphill excursions allowing only perturbations which lead to better solution. In short, when compared to SQ method IFSQ annealing schedule is steeper at the beginning and 


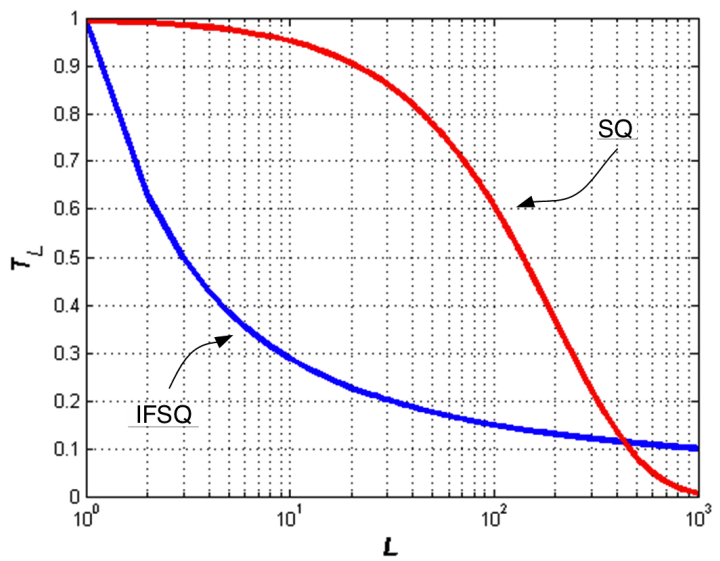

Figure 13. Comparison of standard SQ and IFSQ annealing schedule functions

kept at higher level towards the end of the search which allows higher convergence speed at start up, and better analysis of close neighborhood area at lower temperature levels.

The second important difference between SQ and IFSQ approach is the choice of the probability of uphill movement. The SQ method is based on Boltzmann probability density function with the drawback in its thin tail, which makes the configurations with substantial cost function difference rather unreachable under small number of iterations. It was noted in [17] that Cauchy distribution, which has a fatter body than Boltzmann distribution, permits easier access to larger set of candidates without any significant algorithm convergence loss. The authors define modified Cauchy distribution, which is also used in IFSQ, as:

$$
p(\Delta Q)=\frac{T_{L}}{\left(\Delta Q^{2}+k_{c} T_{L}^{2}\right)^{(D+1) / 2}}
$$

where $k_{c}$ relates $T_{L}$ to $\Delta Q$ and $D$ defines the degrees of freedom. According to eq. 9 parameter $D$ is equal to 20 while modified Cauchy distribution constant $k_{c}$ is chosen to ensure maximum probability of transition $\left(p_{\max }\right)$ at first temperature level $T_{1}$ to be satisfied for $99 \%$ of all possible cost function differences. Since $\Delta Q$ follows the Gaussian distribution $99 \%$ of all possible numerical values of $\Delta Q$ matches three standard deviations $\sigma_{\Delta Q}$ of the corresponding distribution. Hence, $p_{\max }$ can be expressed as:

$$
p_{\text {max }}=\frac{T_{1}}{\left(\left(3 \sigma_{\Delta Q}\right)^{2}+k_{c} T_{1}^{2}\right)^{(D+1) / 2}}
$$

By extracting $k_{c}$ the following statement is obtained:

$$
k_{c}=\sqrt{\frac{1}{T_{1}^{2}}\left(\sqrt[(D+1) / 2]{\frac{T_{1}}{p_{\max }}}-\left(3 \sigma_{\Delta Q}\right)^{2}\right)}
$$

The difference between these two probability transition approaches are seen easily in Figure 14 where transition probability functions of SQ and IFSQ approach are presented. 


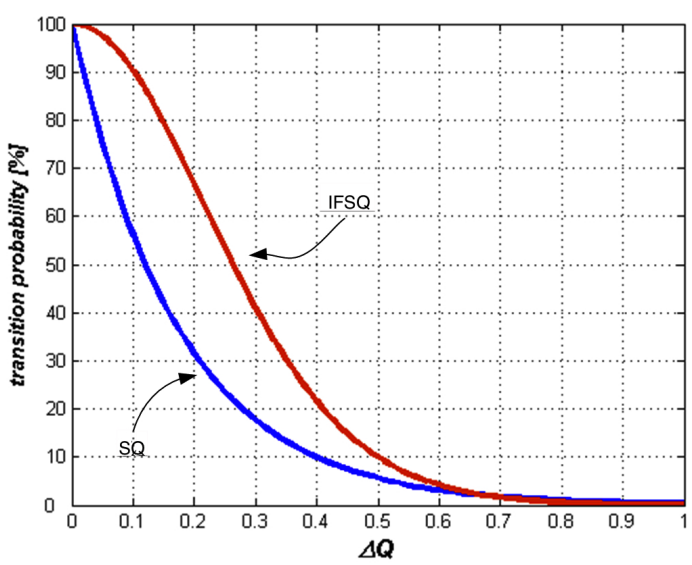

Figure 14. Transition probability functions of SQ and IFSQ approach

As expected the probability function based on modified Cauchy distribution is fatter than the one based on Boltzmann function allowing uphill movements with more ease. The advantage of IFSQ probability transition function lies inside area which covers $\Delta Q$ from 0.1 to 0.6 . With $\Delta Q=0.2$, for example, transition probability of IFSQ marks approximately $65 \%$, while SQ is limited to approximately $32 \%$. B including this middle class of $\Delta Q$ values more often into the search path, search robustness is increased but convergence speed doesn't suffer substantial loss.

The depth of exploration depends not only on the transition probability function but also on the annealing schedule and maximum displacement factor $R_{0}$. The annealing schedule function of IFSQ is very steep at the beginning, suppressing the initial deep exploration rather fast and keeping the rest of the search concentrated on small energy differences around the actual point. Thus, so called deep exploration which easily permits uphill movements is available only at the beginning. Still, these initial search opportunities can not be exploited if $R_{0}$ factor is small as only small part of the potential search space can be reached for the analysis. Small maximum displacement factor is adequate choice for the advanced search status when global minimum area is well located, and extense search is required in order to find the best solution. By keeping $R_{0}$ constant during the entire search process, as in SQ approach, two opposite search requirement can not be met and consequently $R_{0}$ is chosen as their compromise. This is changed in IFSQ approach maximum displacement factor is set to high value covering whole search space at the beginning, and it is gradually decreased as the search advances. The dependence is made according to the:

$$
R_{0}(L)=\delta R_{0}(L-1), R_{0}(0)=r, L=1,2, \ldots
$$

where $L$ stands for the corresponding temperature level, $\delta$ is the search space reduction factor, and $r$ the initial maximum allowed displacement. The numerical values of $\delta$ and $r$ are determined through empirical analysis. Additionally, since IFSQ approach is not oriented only on precision, the maximum number of iterations is not limited to probability, but the search is stopped if in five consecutive temperature levels no cost function improvement is obtained. 


\subsection{Tuning of IFSQ parameters}

The parameters describing IFSQ approach can be divided into the ones inherited from the SQ method and the ones developed for the decoupling solution which correspond to three new IFSQ parameters that have to be set empirically $\left(r, \delta, N_{V C P L}\right)$.

The inherited SQ factors are set to the numerical values obtained in previous SQ analysis while three new IFSQ factors have to be set empirically $\left(r, \delta, N_{V C P L}\right)$. When determining the correct level of the unknown parameters, the parameters that are still not analyzed are over-dimensioned which guarantees the correct functioning of IFSQ search at the cost of execution time. The first set of simulations focuses on adequate pair of parameters for maximum allowed displacement definition $(r, \delta)$. Afterwards, the obtained pair is used for analyzing the appropriate number of visited candidates per temperature level $\left(N_{V C P L}\right)$.

\subsubsection{Search space reduction factor and initial maximum allowed displacement}

Since probability transition function in IFSQ presents high values for all energy differences, it can be stated that IFSQ favors the wide search space exploration during the entire search process. However, the depth of exploration depends not only on the transition probability function but also on the annealing schedule and maximum displacement factor $R_{0}$. The annealing schedule function of IFSQ is very steep at the beginning, suppressing the deep exploration rather fast and keeping the rest of the search concentrated on small energy differences around the actual point.

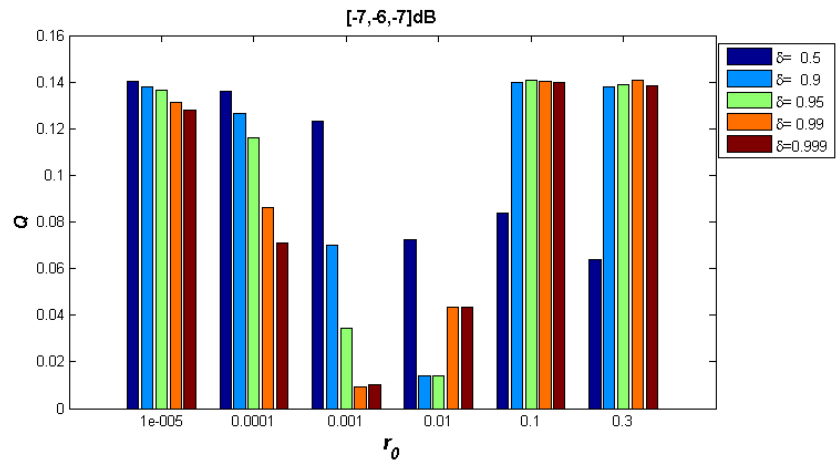

Figure 15. IFSQ search based on different sets of $(r, \delta)$ factors under strong coupling

If the starting search space is large, and the reduction factor very close to one, the search will progress rather slowly. In this case the probability of worse movement decreases and even enters the downhill search but the search space is kept large all the time. As a consequence five consecutive temperature levels without the reduction of cost function occur far before the global minimum area is located. This is confirmed in Figure 15, where the precision of IFSQ search based on different sets of $(r, \delta)$ factors under strong coupling is presented. As is seen in the right image side, when the starting search space is set to $r_{0}=0.1$ or $r_{0}=0.3$ the algorithm presents poor precision for all space reduction factors except for $\delta=0.5$. Still, with reduction factor of $50 \%$ the search is easily drown into saturation as clear tendency path can hardly be established at such fast pace. 
If the starting search space is too small, the search progress cannot move far from the starting point and the obtained solution results depend largely on the coupling strength. Under strong coupling the starting search space corresponding to $r_{0}=10^{-5}$ and $r_{0}=10^{-4}$ represents the selection of narrow starting search space. The obtained cost function precision is generally low, but as the reduction factor gets closer to one, it is increased in both cases. This is logical as if the small search space is additionally reduced at fast pace the algorithm is easily left without any new candidates and no progress can be made.

Consequently, two starting search spaces, in the middle of the image, built around $r_{0}=0.001$ and $r_{0}=0.01$ offer the adequate selection. In fact the best precision under strong coupling is reached with the following four pair of factors: $(0.001,0.99),(0.001,0.999),(0.01,0.90)$ and $(0.01,0.95)$. The pairs are in accordance with the theoretical expectations as they ratify that in vicinity of adequate set of parameters smaller search space requires lower reduction factor to obtain the same precision level.

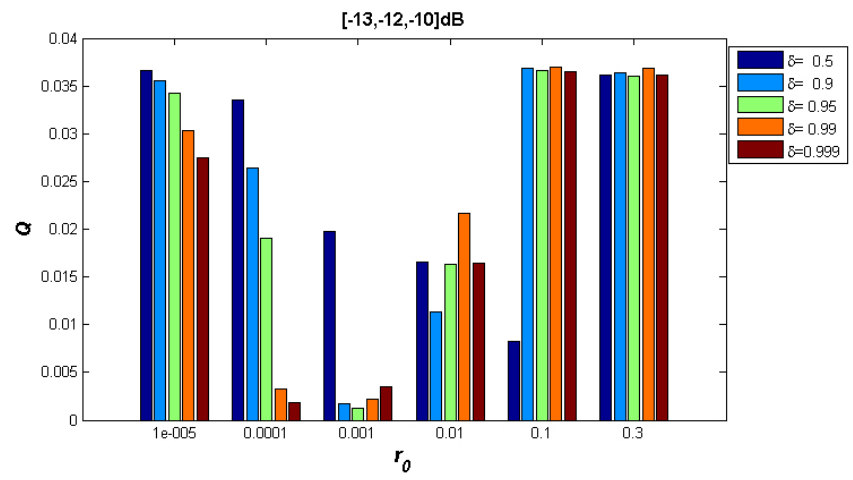

Figure 16. IFSQ search based on different sets of $(r, \delta)$ factors under moderate coupling

The same analysis is repeated for system under moderate coupling of $[13,-12,-10] \mathrm{dB}$ in Figure 16. The behavior of algorithm is similar and the potentially adequate set of parameters is found in the central part of the image. However, in this case the best values are moved slightly to the left since the coupling strength is lower and the the ideal solution is located closer to the initial search point. Comparing the results obtained at two different coupling levels, the only pair of parameters appearing in both is $r_{0}=0.001$ and $\delta=0.99$. Hence, these values are used as adequate parameters for IFSQ search adopted to nonlinear decoupling problem.

\subsubsection{Number of visited points per level}

The number of visited candidates per temperature level defines the number of energy configurations analyzed between two temperature changes. If this number is large, the search space is analyzed in detail which produces stable results but consumes a lot of the processing time. Nevertheless, if the number of visited configurations is small, the search progress is made based on unreliable data which leads to poor cost function results.

The performance of IFSQ search method based on different $N_{V C P L}$ number under strong coupling conditions of $[-8,-6,-6] \mathrm{dB}$ is depicted in Figure 17 . As expected, when $N_{V C P L}$ number is small, like for $N_{V C P L}=5$ or $N_{V C P L}=3$, the search is easily drown into a blind ally. 


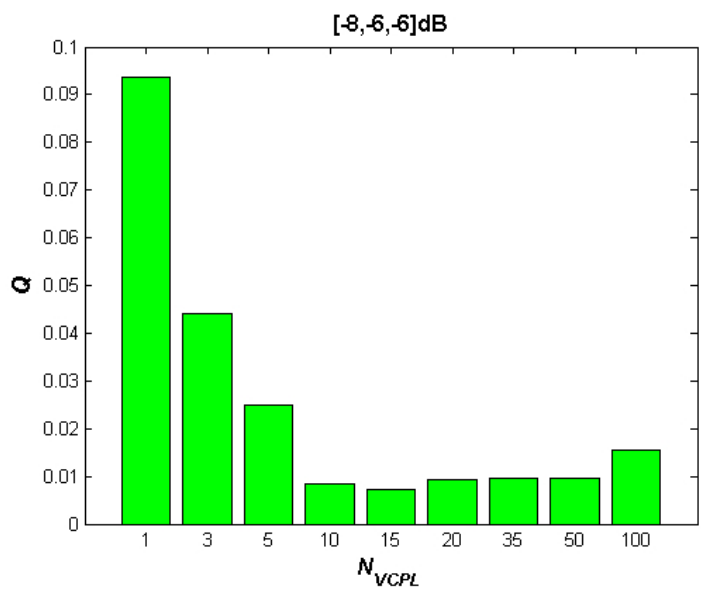

Figure 17. Cost function of IFSQ search with different $N_{V C P L}$ factor under $[-8,-6,-6] \mathrm{dB}$ coupling

Local minimum area is located well, but as all temperature levels are spent fast, no uphill movements are available, and no additional progress can be made despite the additional number of iterations.

On the other hand, if number of visited candidates is large and the temperature reduction function is not smooth enough the search can get stacked while in wandering phase and can only offer local minimum as final solution. Eventually the best precision under strong coupling is reached with $N_{V C P L}=10$ and $N_{V C P L}=15$.

When the analysis is centered on moderate coupling the behavior of IFSQ method changes very little and the adequate selection of visited number of candidates per level lies between 5 and 15. Since strong coupling conditions discard $N_{V C P L}=5$ as too small, and moderate coupling exhibits slightly better results with $N_{V C P L}=10$ than with $N_{V C P L}=15$, the adequate parameter adjusted to the nonlinear coupling problem is set to $N_{V C P L}=10$.

\subsection{IFSQ example}

The parameters of IFSQ algorithm are adjusted to nonlinear coupling problem through empirical analysis based on different set of simulations. The adopted set of IFSQ search parameters is presented in Table 3. The table consists of two columns with inherited SQ values in the left column, and new IFSQ functions and parameters values based on empirical analysis in the right one.

The behavior of IFSQ algorithm applied to coupling of [-10,-12,-12]dB is shown in Figure18, where cost function is presented in dependence of number of iterations for 10 instances of IFSQ search under same operating conditions.

Unlike SQ search which produced 10 closely related curves, the IFSQ curves are spread over wider area as the search progresses due to the higher influence of probability since precision is sacrificed in favor of smaller number of iterations.

Furthermore, the IFSQ cost function is also based on quadratic mean square function for which it has been demonstrated in [1] that smaller $Q$ is not transformed into any significant 


\begin{tabular}{|c|c|}
\hline Inherited SQ Values & New IFSQ Functions \\
\hline $\operatorname{Res}_{\text {symb }}=0.00001$ & $\begin{array}{c}T_{L}=\frac{T_{\alpha}}{\ln (L+1)} \\
T_{\alpha}=0.69, L=1,2,3 \ldots\end{array}$ \\
\hline $\operatorname{Res}_{\text {coeff }}=0.00001$ & $p(\Delta Q)=\frac{T_{L}}{\left(\Delta Q^{2}+k_{c} T_{L}^{2}\right)^{(D+1) / 2}}$ \\
\hline $\operatorname{Coef}_{0}(i)=\left\{\begin{array}{l}1, \quad i=1 \\
0, i=2, \ldots, 20\end{array}\right.$ & $k_{c}=\sqrt{\frac{1}{T_{1}^{2}}\left(\sqrt[(D+1) / 2]{\frac{T_{1}}{p_{\max }}}-\left(3 \sigma_{\Delta Q}\right)^{2}\right)}$ \\
\hline $\begin{array}{c}\operatorname{Coef}_{\text {new }}(i)=\xi_{i} R_{0}+\operatorname{Coef}_{a c t}(i) \\
i=1 . .20 ; \xi_{i} \in\langle-1,1\rangle\end{array}$ & $\begin{array}{l}R_{0}(L)=\delta R_{0}(L-1) \\
R_{0}(0)=r, L=1,2, \ldots\end{array}$ \\
\hline$Q=\sqrt{\frac{1}{N} \sum_{i=1}^{N}\left(x_{1 i}-\hat{x}_{1 i}\right)^{2}}$ & $\begin{array}{c}r=0.001 \\
\delta=0.99\end{array}$ \\
\hline \multirow[t]{2}{*}{$p_{\max }=50 \%$} & $N_{V C P L}=10$ \\
\hline & $Q_{\text {suff }}=0.005$ \\
\hline
\end{tabular}

Table 3. Theoretic set of IFSQ search parameters after empirical analysis

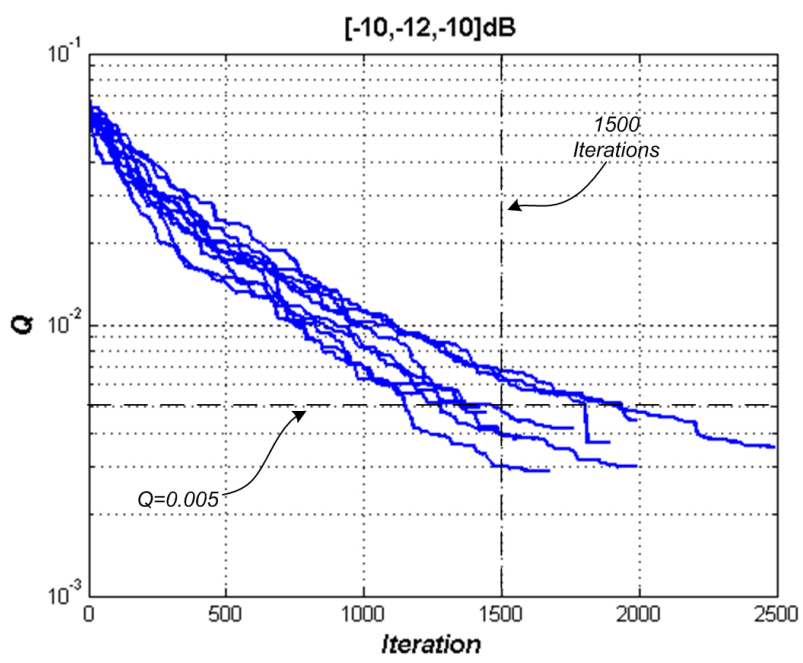

Figure 18. Ten instances of adjusted IFSQ search under moderate coupling

BER enhancement for $Q<0.005$. Since IFSQ is not oriented only on precision of search can be stopped when the cost function reaches $Q$ limit. This occurs on average for 1500 number of iterations as shown in Figure 19 where the corresponding cost function value after 1500 iteration is presented for all ten search instances. The average of all ten executions is located at $\bar{Q}=0.0056$ which indicates that the search under moderate coupling can be suspended when 1500 iterations are surpassed. When compared with 4000 iterations that is required to SQ approach to reach $Q=0.005$, this presents the reduction of processing load of approximately $37 \%$. When compared to the SQ approach which is not suspended at some cost function limit, the processing gain is even higher as it is reduced from approximately 16000 iterations to 1500 corresponding to reduction of more than $90 \%$. Eventually, if after 1500 iterations the quality of the result is far from $Q=0.005$ the search can easily be repeated with the consequent slight reduce of the processing load gain. 


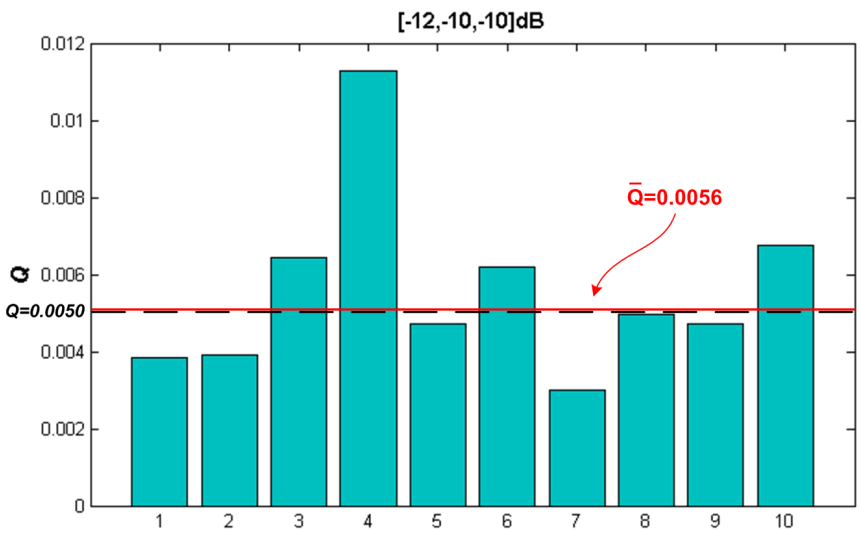

Figure 19. The precision of IFSQ search after 1500 iterations

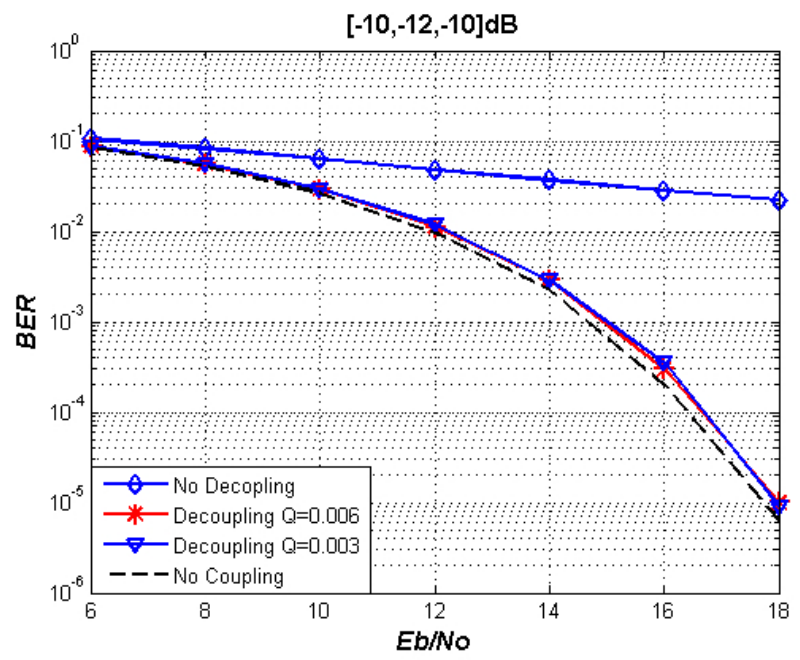

Figure 20. Performance of IFSQ based decoupling module under moderate coupling

The reduction of permitted number of iterations to 1500 is verified in Figure 20 where the performance of IFSQ decoupling module under moderate coupling conditions is presented. The first curve on the image presents the behavior of system without the decoupling module under coupling of $[-10,-12,-10] \mathrm{dB}$. The lowest curve shows the theoretical system performance with only AWGN. Two curves in the middle present two decoupling module instances based on IFSQ approach stopped after 1500 iterations. Actually, the coefficients used for generating this two curves correspond to the seventh and tenth IFSQ execution depicted in Figure 19. Regardless of the numerical difference of cost functions, the performance of both decoupling modules is very similar. Since the search is abandoned when cost function reaches sufficient $Q$ value defined with $Q_{s u f f}=0.005$ the performance of decoupling module does not follow the theoretical transmission curve as close as is the SQ approach. 


\section{Conclusion}

The effect of coupling are minimized with the software module positioned at the output of analog-to-digital converter and before any further digital signal processing. Inside the module, signal reconstruction based on the approximation of inverse nonlinear coupling is achieved. The generation of inverse coupling function is based on fitting surface approach which can be divided into two steps: calibration process and point-to-point real-time decoupling. Inside calibration process inverse nonlinear coupling surface is build based on function formed with 20 degrees of freedom. Inspired by SA class of approaches, two SQ algorithms are proposed for locating the optimal inverse function parameters.

Even though classical SQ method can not be used under real time conditions it provides the upper limit of the decoupling precision module and is used for tuning the required system parameters like for example calibration data size. It is intended only for proof of concept and precision reference to other two decoupling search approaches. Eventually actually confirms the adequate selection of the surface model and proves the concept of decoupling software module. The performance of decoupling module based on the SQ search method shows stable behavior and excellent decoupling results.

The proposed Improved Fast SQ search method is included in decoupling module as the fast version of the previously developed SQ search. It follows the same search logic as the SQ algorithm with the difference in the annealing schedule which is much steeper, the initial search space is wider and the probability of uphill movement is higher at the beginning. This way the search is carried out more thoroughly in the initial stage, allowing stepper search progress sooner than in the original SQ approach. Furthermore, since the search space is reduced according to the exponential function, the maximum allowed displacement is reduced with temperature, and as consequence the search concentrates more on small space around the global minimum area. The performance of IFSQ decoupling module, based on 1500 iterations, shows good decoupling results, with slightly lower BER level when compared to the standard SQ search. Nevertheless, the required number of iterations is reduced by more than $90 \%$ which justifies the precision loss. The approach requires less processing power than $\mathrm{SQ}$, and as such might be used in mobile phones inside the decoupling module.

With the introduction of normalization, the search domain is well defined between $\langle-1,1\rangle$, and the search depth is easily controlled with symbol resolution factor. This proposal allows the adaptation of implemented search methods to general decoupling problem offering at the same time constant precision and efficient optimization of search process with no restrictions on the physical coupling source nor signal constellation.

\section{Acknowledgments}

This work has been carried out in the frame of Spanish MCIN project TEC2009-14219-C03-01.

\section{Author details}

Igor Arambasic, Javier Casajus Quiros and Ivana Raos

ETSI Telecomunicacion, Universidad Politecnica de Madrid, Spain 


\section{References}

[1] Arambasic, I. [2008]. RF Front-End Non-Linear Coupling Cancellation, PhD thesis, Escuela Técnica Superior de Ingenieros de Telecomunicación.

[2] Arambasic, I., Quiros, F. J. C. \& Raos, I. [2007a]. Efficient rf front-end non-linear multi antenna coupling cancellation techniques, PIMRC 2007. IEEE 18th International Symposium on Personal, Indoor and Mobile Radio Communications, 2007 pp. $1-5$.

[3] Arambasic, I., Quiros, F. J. C. \& Raos, I. [2007b]. Improvement of multiple antennas diversity systems through receiver nonlinear coupling cancellation, 2007. International Waveform Diversity and Design Conference pp. $102-106$.

[4] Drago, G., Manella, A., Nervi, M., Repetto, M. \& Secondo, G. [1992]. A combined strategy for optimization in non linear magnetic problems using simulated annealing and search techniques, IEEE Transactions on Magnetics 28(2): 1541-1544.

[5] Finnerty, S. \& Sen, S. [2004]. Simulated annealing based classification, Sixth International Conference on Tools with Artificial Intelligence, 1994. Proceedings. (Nov): $824-827$.

[6] Forrest, S. [1996]. Genetic algorithms, Computing Surveys 28: 77-80.

[7] Ingber, L. [1989]. Very fast simulated re-annealing, Lester Ingber Papers 89vfsr, Lester Ingber. available at http:/ /ideas.repec.org/p/lei/ingber/89vfsr.html.

[8] Ingber, L. [1993]. Simulated annealing: Practice versus theory, Mathl. Comput. Modelling 18(11): 29-57.

[9] Ingber, L. [1996]. Adaptive simulated annealing (asa): Lessons learned, Lester Ingber Papers 96as, Lester Ingber. available at http://ideas.repec.org/p/lei/ingber/96as.html.

[10] Ingber, L. \& Rosen, B. [1992]. Genetic algorithms and very fast simulated reannealing: a comparison, Mathematical and Computer Modelling pp. 87-100.

[11] Johnson, D. S., Aragon, C. R., McGeoch, L. \& Schevon, C. [1989]. Optimization by simulated annealing: An experimental evaluation, Operations Research pp. 865-892.

[12] Kirkpatric, S., Galett, C. D. \& Vecchi, M. P. [1983]. Optimisation by simulated annealing, Science 220(4598): 621-680.

[13] Li, Y., Yao, J. \& Yao, D. [2002]. An efficient composite simulated annealing algorithm for global optimization, IEEE 2002 International Conference on Communications, Circuits and Systems and West Sino Expositions 21: 1165- 1169.

[14] Mendonca, P. \& Caloba, L. [1997]. New simulated annealing algorithms, Proceedings of 1997 IEEE International Symposium on Circuits and Systems, 1997. ISCAS '97. 3: 1668-1671.

[15] Renyuan, T., Shiyou, Y., Yan, L., Geng, W. \& Tiemin, M. [1996]. Combined strategy of improved simulated annealing and genetic algorithm for inverse problem, IEEE Transactions on Magnetics 32: 1326 - 1329.

[16] Rosen, B. [1992]. Function optimization based on advanced simulated annealing, Workshop on Physics and Computation, 1992 pp. 289-293.

[17] Szu, H. H. \& Hartley, R. L. [1987]. Nonconvex optimization by fast simulated annealing, Proceedings of the IEEE 75(11): $1538-1540$.

[18] van Laarhoven, P. J. M. \& Aarts, E. [1987]. Simulated Annealing: Theory and Applications, D. Reidel. 